Ahmed, Bakhtiyar, Dannhauser, Thomas and Philip, Nada (2019) A systematic review of reviews to identify key research opportunities within the field of eHealth implementation. Journal of Telemedicine and Telecare, 25(5), pp. 276-285. Copyright (C) 2018. Reprinted by permission of SAGE Publications. 


\title{
A systematic review of reviews to identify key research opportunities within the field of eHealth implementation
}

\section{Bakhtiyar Ahmed ${ }^{1}$, Thomas Dannhauser² and Nada Philip'}

\begin{abstract}
This paper is a systematic review of the relevant literature surrounding the implementation and utilisation of eHealth in order to identify key challenges and opportunities to future eHealth applications. NHS Evidence, PubMed, IEEE Explorer, Cochrane Library and JMIR Publications were all searched for reviews published between the dates 1 January 2010 and 30 June 2017. 47 papers met the final inclusion criterion. The published literature focused on a wide array of challenges, categorised into five areas, facing the implementation and utilisation of eHealth and from this, four areas of opportunity to advance eHealth were identified. The five challenge areas are (C1) Stakeholders and System Users, (C2) Technology and Interoperability, (C3) Cost-Effectiveness and Start-up Costs, (C4) Legal Clarity and Legal Framework and (C5) Local Context and Regional Differences. The four opportunity areas are (O1) Participation and Contribution, (O2) Foundation and Sustainability, (O3) Improvement and Productivity and (O4) Identification and Application. The literature analysed in this systematic review identifies design and implementation priorities that can guide the development and utilisation of future eHealth initiatives.
\end{abstract}

\section{Keywords}

Challenges, eHealth, Implementation, Opportunities, Utilisation

\footnotetext{
${ }^{1}$ Kingston University London, UK

${ }^{2}$ University College London, UK

Corresponding author:

Bakhtiyar Ahmed, Digital Media for Healthcare (DigHealth), Kingston University London, Penrhyn Road, Kingston upon Thames, Surrey, KT1 2EE, UK.

Email: b.ahmed@kingston.ac.uk
} 


\section{Introduction}

eHealth is a rapidly growing area distinguished by the utilisation of information technology and electronic communication for the transmission, storage and retrieval of digital data for administrative, educational and clinical purposes. eHealth is considered by many as a broad term containing a wide variety of digital health subdomains ranging from Electronic Health/Medical Records (EHR/EMR) to virtual health to big data health systems, etc. The use of eHealth technologies is spreading globally and is thus supporting health and prosperity for individuals all around the world [1]. In recent times, despite many eHealth projects failing to be implemented (regardless of the extensive research and availability of literature in the field), several eHealth tools and services have still been introduced and deployed in order to perform key health-related functions effectively and efficiently [2]. Amongst the many potential benefits of eHealth is its ability to provide efficient, convenient and cost-effective delivery of care as well as decision making that is more informed [3]. Available findings indicate that evidence-based eHealth procedures are key to delivering high-quality care and healthcare service efficiency [1]. Consequently, there appears to be great potential for eHealth to deliver individuals affordable healthcare without compromising on the quality of service [4]. Some of the most promising and noticeably established applications of information technology today are in the field of eHealth.

Accordingly, there has been an increase in the number of studies evaluating the effectiveness of eHealth implementation and utilisation. Many of these studies discuss the problems faced throughout the project and product development life cycle and go on to provide recommendations for future actions along with the lessons learnt. Resultantly, much has been learnt about the challenges of employing new eHealth technology into everyday clinical practice. However, to the best of these authors' knowledge, there has been no work in the field, which identifies and categorises the future actions and lessons learnt, etc. mentioned within these papers i.e. opportunistic areas of eHealth research and development. This, in turn makes it difficult for eHealth projects to determine where to focus their efforts on before embarking on the application of a new eHealth solution [2], [4]. As eHealth continually evolves, so do the challenges to its application, as time progresses it is evident that there will be new and different challenges faced by eHealth projects when implementing and utilising eHealth [3]. This makes it essential for there to be work in the field which builds upon and updates (rather than repeats) existing literature thus guiding future work. As eHealth is considered a broad term, it is important to cover implementation and utilisation challenges and opportunities from all perspectives of eHealth. Resultantly, this review will not limit itself to any subdomain or application of eHealth.

One of the main aims of this review is to consider any existing and new challenges (identified after the year 2010), of eHealth implementation and utilisation. This thereby provides readers with an up to date insight into the various kinds of hurdles faced by modern eHealth projects. Another aim, which is novel work in the field, is to identify and categorise any recommendations, i.e. future actions along with lessons learnt, etc. mentioned within these papers and subsequently recognise potential opportunities that can be studied, to explore priority eHealth research and development areas. To achieve these aims, a systematic review of reviews was conducted on the theme of eHealth challenges and opportunities, focusing specifically on papers relating to the implementation and utilisation of eHealth ranging from but not limited to key areas of eHealth activity such as personnel management, design considerations and existing/new technologies and services, etc. This systematic review firstly identifies all the challenges mentioned within each paper before categorising these into one of five areas. Secondly, the papers are reviewed once again, but this time identifying any opportunistic areas mentioned within each paper, 
which are then categorised into one of four areas. The review will then go on to discuss each of these nine areas in detail before concluding.

The review aims to answer the following questions:

(i) What are the recent challenges of eHealth implementation and integration?

(ii) What are the key opportunities that arise from these challenges of eHealth implementation and integration?

(iii) What can we learn about the effectiveness and efficacy of eHealth implementation best practices and research?

(iv) Finally, what do we have to learn in order to maximise eHealth utilisation for future solutions?

\section{Methods}

To identify relevant studies, narrative reviews, systematic reviews, meta-syntheses and metaethnographies were all considered. The bibliographic databases examined in the search for relevant academic published articles were NHS Evidence, PubMed, IEEE Explorer, Cochrane Library and JMIR Publications. It is acknowledged that as with most systematic reviews, the search methods may not identify all relevant literature. However, to minimise this risk there will be an inclusive search strategy. The inclusion criteria used:

- the following search terms relating to (1) eHealth: eHealth and e-health and

- the following search terms relating to (2) challenges and opportunities: barrier*, challeng*, facilitat*, fail*, inhibit*, opportunit*, promot* and *success*

- both notions (1) and (2) were then combined before limiting the search results by identifying the search terms in paper titles, abstract, keywords, publication language, publication date and lastly publication type.

In relation to the exclusion criteria;

- studies that were not in the English language,

- not between 01/01/2010 to 30/06/2017 along with

- studies that were not considered as journal articles, i.e. comparative studies, evaluation studies, editorials or comments were all excluded.

The flowchart of study selections is shown in Figure 1. To ensure that the above inclusion criteria were met, two reviewers examined the full texts of all the identified manuscripts. If the reviewers could not exclude a paper based on its abstract, the full paper was obtained. 47 papers were identified and agreed upon by both reviewers to have met the inclusion criteria. All papers were double screened and cross-reviewed by both reviewers to ensure that data were appropriately categorised. In case of disagreement over the categorisation of data, both reviewers would read the paper together once more and through discussion come to an agreement. Data were then extracted and tabled from these 47 manuscripts and categorised in terms of the challenges and opportunities identified within each paper. This thematic analysis involved listing all identified challenges and opportunities before grouping them into categories based on similar characteristics, again this was done by both authors jointly. More details of the categorisation process are discussed in the Results section below. Next, a detailed qualitative analysis of the challenges and opportunities was conducted to elaborate upon specific issues influencing the implementation and utilisation of eHealth. 


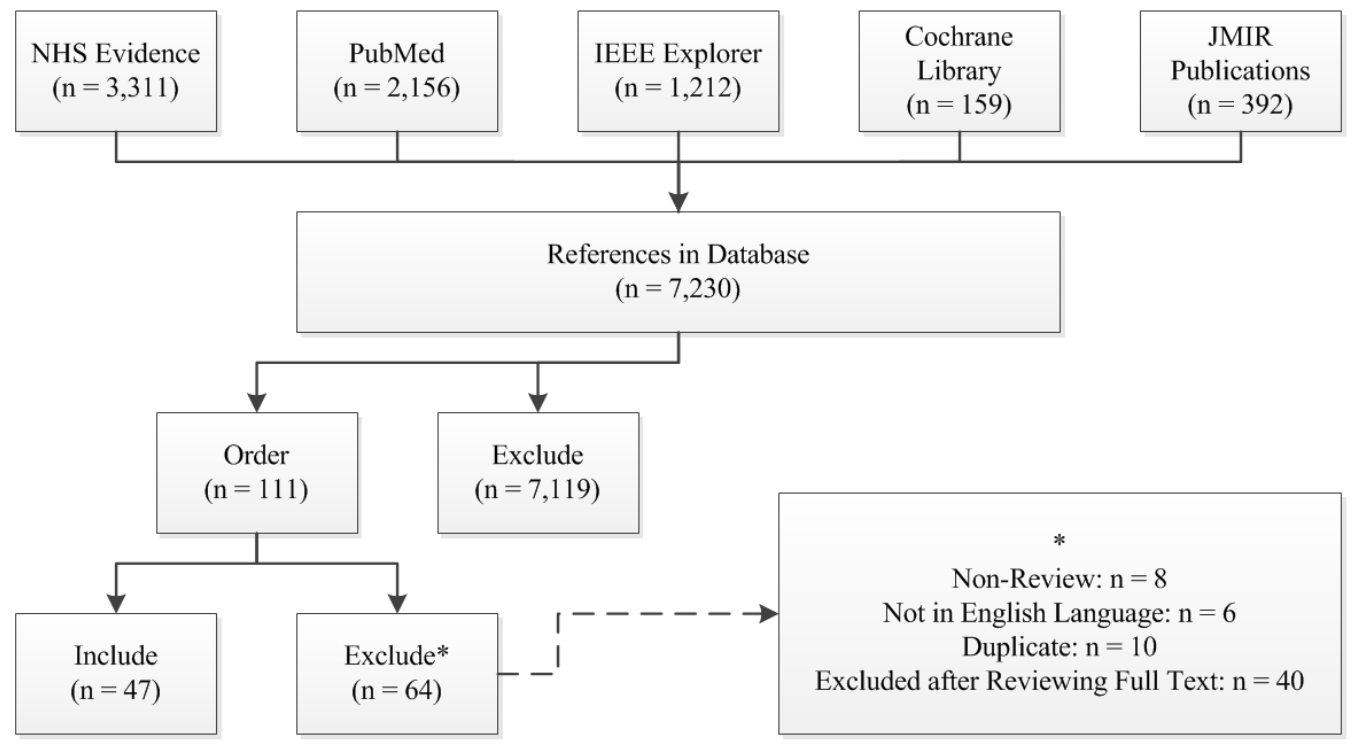

Figure 1. Flowchart of study selection.

\section{Results}

From 7,320 citations screened, 111 met the above inclusion criteria. Of these:

- eight were non-reviews,

- six were not in the English language,

- 10 were duplicates and

- 40 were excluded after a full-text review revealed that these were either not specifically on eHealth, its implementation or on its utilisation.

This left 47 papers that did meet the inclusion criteria. Of these 47:

- 11 were published between 2010 and 2011,

- 15 between 2012 and 2014, 10 in 2015 and

- 11 between 2016 and June of 2017.

Challenges to the implementation and utilisation of eHealth were classified into one of five areas (Figure 2). These areas were identified by grouping challenges into categories based on recurring concepts and themes. Once identified, these areas were given suitable names which were associated with their major characteristics. The details and the characteristics by which each category was identified is as follows: 
- C1: Stakeholders and System Users (78.7\%; $n=37 / 47)$ - Many papers discussed challenges relating to the wide array of personnel involved with eHealth projects e.g. patients, healthcare professionals, etc.,

- C2: Technology and Interoperability (66.0\%; $n=31 / 47)$ - Two-thirds of papers discussed challenges relating to the various aspects of technologies involved in eHealth projects such as integrity of data, security concerns, etc.,

- C3: Cost-Effectiveness and Sustainability (21.3\%; $n=10 / 47)$ - Several papers discussed challenges relating to the capital involved in eHealth projects, such as acquiring funding to costs associated with on-going maintenance, etc.,

- C4: Legal Clarity and Legal Framework (21.3\%; $n=10 / 47)$ - Several papers discussed challenges relating to the legalities surrounding eHealth projects such as privacy concerns and existing/upcoming laws, etc

- C5: Organisational Context and Regional Differences (53.2\%; $n=25 / 47$ ) - More than half of papers discussed challenges relating to the settings in which eHealth projects are applied, these settings range from within the hospital site to a country-wide context

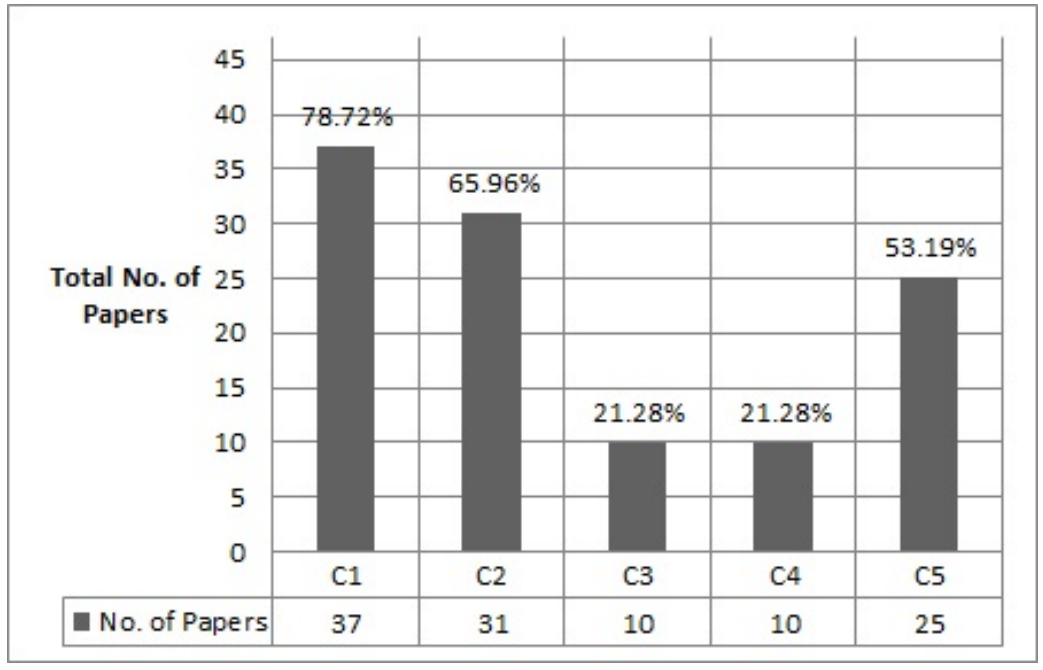

Figure 2. Number and percentage of papers identifying challenges categorised, into each challenge area.

Like the categorisation of challenges, opportunities were grouped together into one of four areas (Figure 3). As the focus of this review is on the implementation and utilisation of eHealth, it was deemed fitting to base these areas around the key stages of a project life cycle i.e. analysis, design and implementation. However, due to the breadth of studies focussing on the implementation of eHealth solutions, this phase was further divided between opportunities discussing existing solutions and opportunities discussing future solutions. 
- O1: Participation and Contribution (55.3\%; $n=26 / 47$ ) - Many papers discussed opportunities relating to the initiation and analysis phases of eHealth projects

- O2: Foundation and Sustainability $(51.1 \% ; n=24 / 47)$ - More than half of papers discussed opportunities relating to the design phase of eHealth projects

- O3: Improvement and Productivity $(46.8 \% ; n=22 / 47)$ - Several papers discussed opportunities relating to the implementation and deployment phases of existing eHealth projects

- O4: Identification and Application (55.3\%; $n=26 / 47)$ - Many papers discussed opportunities relating to the implementation and deployment phases of future eHealth projects

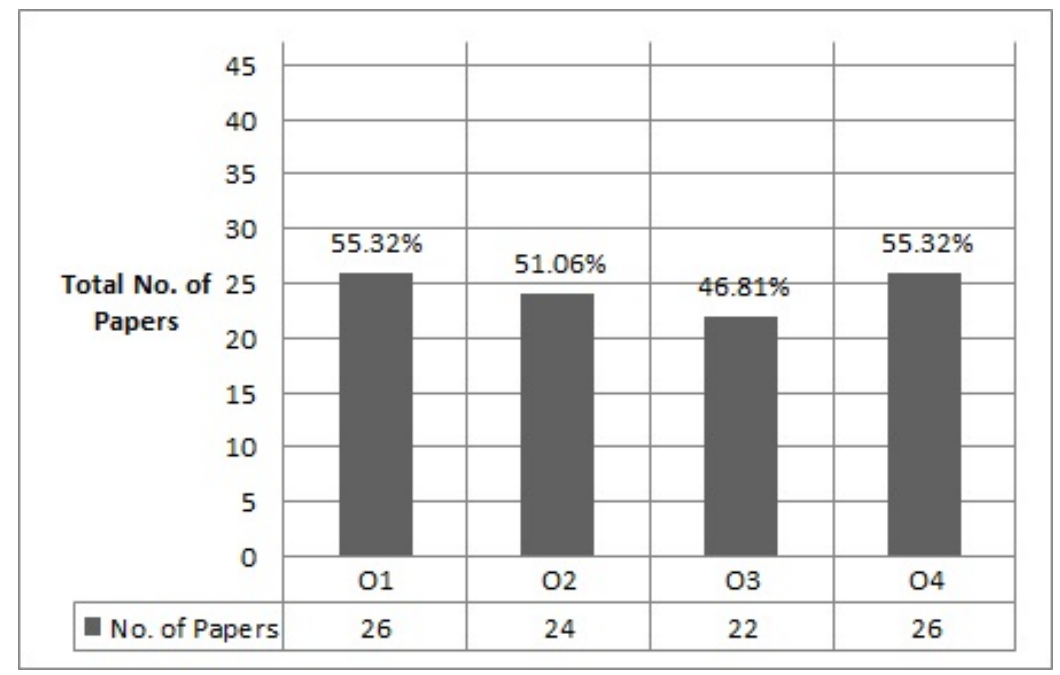

Figure 3. Number and percentage of papers identifying opportunities, categorised into each opportunity area.

The results of the categorisation of these 47 manuscripts are detailed in Table 1 below.

\section{Discussion}

This section will elaborate upon and discuss the individual challenges and opportunities identified within the analysed set of literature. 


\begin{tabular}{|c|c|c|c|c|c|c|c|c|c|c|c|c|c|c|c|c|c|}
\hline \multirow{4}{*}{\multicolumn{2}{|c|}{ 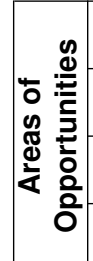 }} & Oे & & & & & $\times$ & $\times$ & $\times$ & $\times$ & & & & $\times$ & $\times$ & & \\
\hline & & Oొ & & & & $\times$ & & $\times$ & & $\times$ & $\times$ & & & & $\times$ & & \\
\hline & & ชิ & $\times$ & $\times$ & $\times$ & & & & $\times$ & & & $\times$ & & & & $\times$ & $\times$ \\
\hline & & $\overline{0}$ & $\times$ & $\times$ & $\times$ & & $\times$ & $\times$ & & & $\times$ & $\times$ & $\times$ & $\times$ & & $\times$ & $\times$ \\
\hline \multirow{5}{*}{\multicolumn{2}{|c|}{ 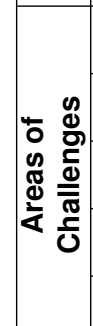 }} & S̊ & $\times$ & $\times$ & $\times$ & $\times$ & $\times$ & $\times$ & $\times$ & & & $\times$ & $\times$ & & & & $\times$ \\
\hline & & ঠ & & & $\times$ & & $\times$ & & & & & & & & & & \\
\hline & & $\mathscr{J}$ & & & $\times$ & & $\times$ & & $\times$ & & & & & & & & \\
\hline & & ชิ & & & $\times$ & & $\times$ & $\times$ & & & $\times$ & $\times$ & & $\times$ & & & $\times$ \\
\hline & & $\overline{0}$ & $\times$ & $\times$ & $\times$ & $\times$ & $\times$ & $\times$ & $\times$ & $\times$ & $\times$ & $\times$ & $\times$ & $\times$ & $\times$ & $\times$ & \\
\hline \multirow{2}{*}{\multicolumn{2}{|c|}{$\begin{array}{l}\frac{\overline{0}}{0} \\
\frac{0}{0} \\
\frac{0}{0} \\
0 \\
\frac{0}{0} \\
\frac{0}{0}\end{array}$}} & 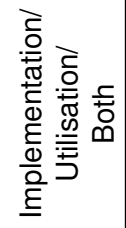 & 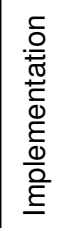 & $\begin{array}{l}\text { 동 } \\
\text { Ф }\end{array}$ & 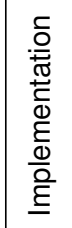 & 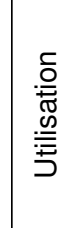 & 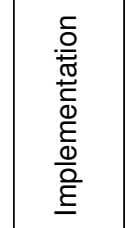 & 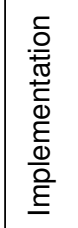 & 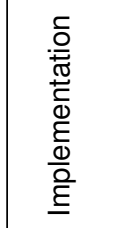 & 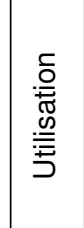 & 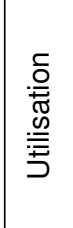 & 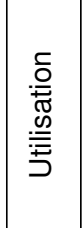 & 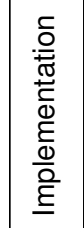 & 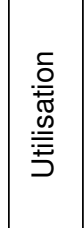 & 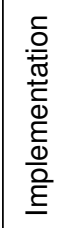 & 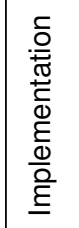 & 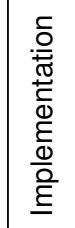 \\
\hline & & 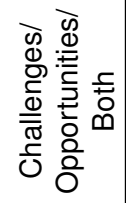 & 阜 & $\begin{array}{l}\text { 동 } \\
\text { Ф }\end{array}$ & 동 & 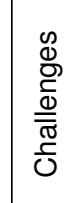 & 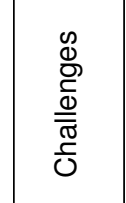 & 喆 & 홈 & F & 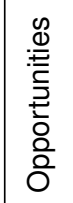 & $\begin{array}{l}\text { 듬 } \\
\text { Ф }\end{array}$ & $\begin{array}{l}\text { 동 } \\
\text { Ф }\end{array}$ & $\begin{array}{l}\text { Fे } \\
\stackrel{0}{\infty}\end{array}$ & 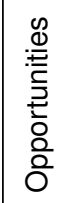 & 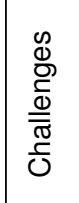 & 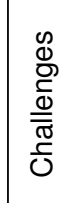 \\
\hline \multirow[b]{2}{*}{ 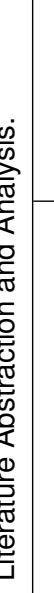 } & & 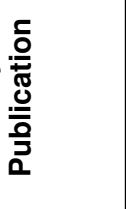 & 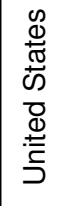 & $\begin{array}{l}\frac{\pi}{0} \\
\frac{\pi}{\frac{\pi}{2}} \\
\frac{\overline{0}}{\overline{0}} \\
\infty \\
\infty\end{array}$ & $\begin{array}{l}\text { 믐 } \\
\stackrel{\underline{\sigma}}{\Phi} \\
\underline{\underline{D}}\end{array}$ & 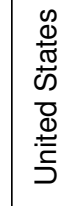 & $\frac{\frac{\pi}{\omega 0}}{\frac{\pi}{\pi}}$ & $\frac{\varepsilon}{\frac{E}{D}}$ & $\frac{2}{\frac{2}{0}}$ & 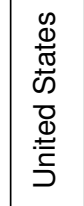 & 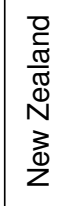 & 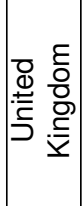 & 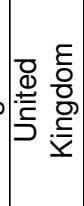 & 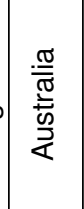 & 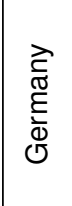 & 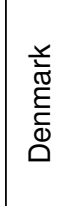 & 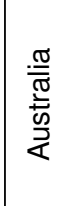 \\
\hline & & 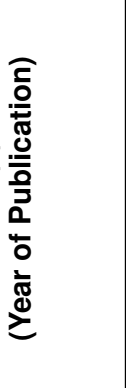 & 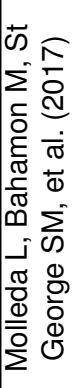 & 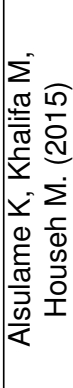 & 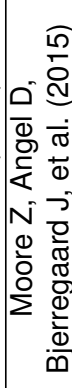 & 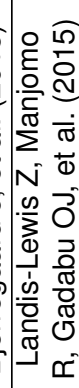 & 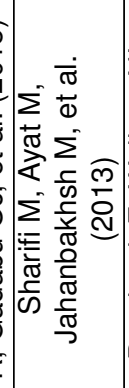 & 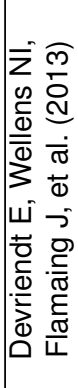 & 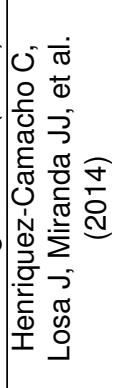 & 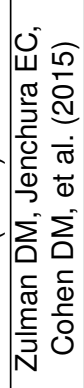 & 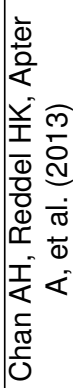 & 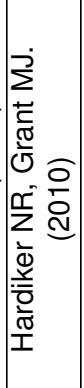 & 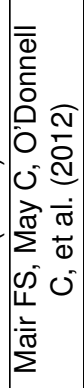 & 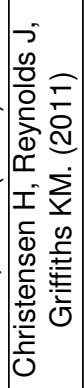 & 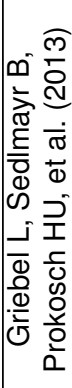 & 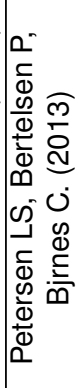 & 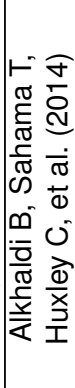 \\
\hline & & & $\underline{\Omega}$ & $\bar{\theta}$ & $\Sigma$ & $\Phi$ & $\sigma$ & 을 & $\Xi$ & $\stackrel{\widetilde{N}}{\underset{\Sigma}{2}}$ & $\stackrel{m}{\Xi}$ & $\underset{\Xi}{\Xi}$ & $\stackrel{\square}{\check{D}}$ & $\underset{v}{\square}$ & $\underset{\Xi}{\Sigma}$ & $\stackrel{\infty}{\square}$ & $\bar{\sigma}$ \\
\hline
\end{tabular}




\begin{tabular}{|c|c|c|c|c|c|c|c|c|c|c|c|c|c|c|c|c|c|}
\hline$\times$ & & $\times$ & & $\times$ & $\times$ & $\times$ & $\times$ & $\times$ & $\times$ & $\times$ & & & $\times$ & $\times$ & & $\times$ & \\
\hline & & $\times$ & & & & & $\times$ & $\times$ & $\times$ & $\times$ & $\times$ & & & $\times$ & $\times$ & & \\
\hline$\times$ & & $\times$ & $\times$ & $\times$ & & $\times$ & & & $\times$ & & $\times$ & $\times$ & & $\times$ & $\times$ & & $\times$ \\
\hline & $\times$ & & $\times$ & $\times$ & & & & & & & & $\times$ & $\times$ & & $\times$ & & $\times$ \\
\hline$\times$ & $\times$ & $\times$ & & & & $\times$ & & $\times$ & $\times$ & & $\times$ & $\times$ & $\times$ & & & & $\times$ \\
\hline & $\times$ & & $\times$ & $\times$ & $\times$ & & & & & & & & & $\times$ & & $\times$ & $\times$ \\
\hline & & & & $\times$ & & & & & & $\times$ & $\times$ & $\times$ & $\times$ & & & & $\times$ \\
\hline$\times$ & $\times$ & $\times$ & $\times$ & $\times$ & $\times$ & $\times$ & $\times$ & $\times$ & $\times$ & & & & $\times$ & & $\times$ & $\times$ & $\times$ \\
\hline & $\times$ & & $\times$ & $\times$ & & & $\times$ & $\times$ & $\times$ & $\times$ & & $\times$ & $\times$ & $\times$ & $\times$ & & $\times$ \\
\hline 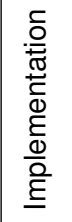 & 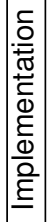 & 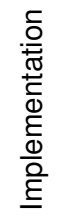 & 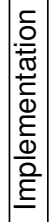 & 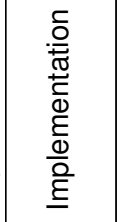 & 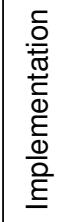 & 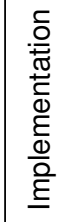 & 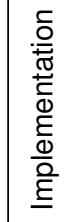 & 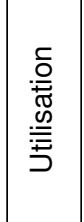 & 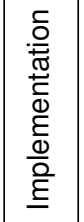 & 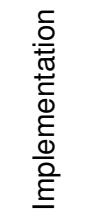 & 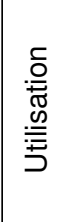 & 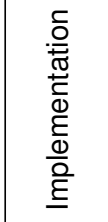 & 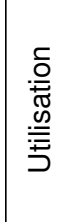 & $\begin{array}{l}\text { Fै } \\
\text { D }\end{array}$ & $\begin{array}{l}\text { Fे } \\
\text { ○ }\end{array}$ & 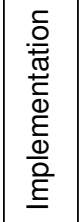 & 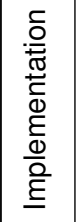 \\
\hline $\begin{array}{l}\text { 호 } \\
\text { Ф }\end{array}$ & 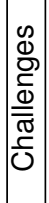 & $\begin{array}{l}\text { 돔 } \\
\text { }\end{array}$ & 壱 & 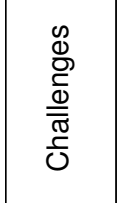 & 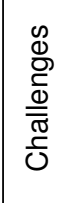 & 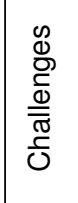 & 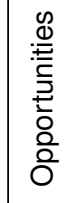 & 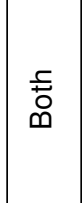 & $\begin{array}{l}\text { 호 } \\
\text { Ф }\end{array}$ & 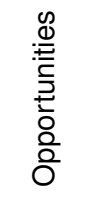 & 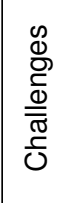 & 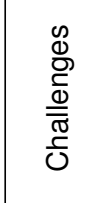 & 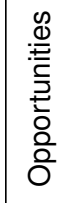 & 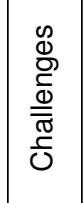 & $\begin{array}{l}\text { Fे } \\
\text { Фे }\end{array}$ & 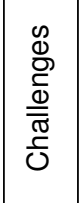 & 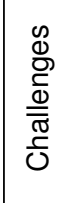 \\
\hline 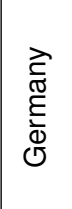 & 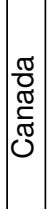 & 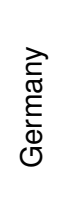 & 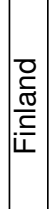 & $\begin{array}{l}\frac{}{0} \\
\frac{0}{0} \\
3 \\
\omega\end{array}$ & : & & 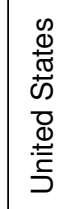 & 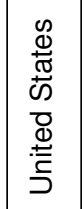 & 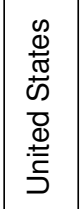 & 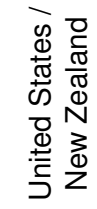 & $\begin{array}{l}\stackrel{\sigma}{0} \\
\underline{\underline{C}}\end{array}$ & 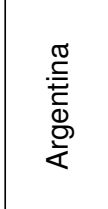 & 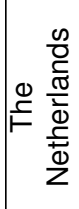 & $\begin{array}{l}\frac{\pi}{\frac{\pi}{\sigma}} \\
\frac{\partial}{\omega}\end{array}$ & 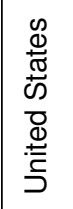 & $\begin{array}{l}\mathbb{\Xi} \\
\stackrel{0}{\pi} \\
\frac{\pi}{4}\end{array}$ & $\begin{array}{l}\sum_{0}^{\overrightarrow{0}} \\
\text { z }\end{array}$ \\
\hline 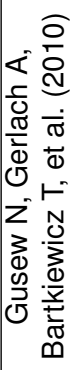 & 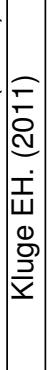 & 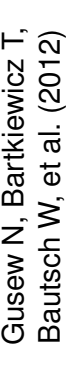 & 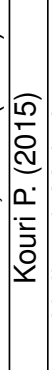 & 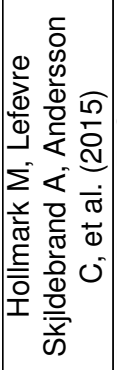 & 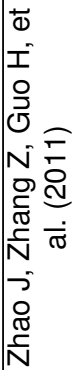 & 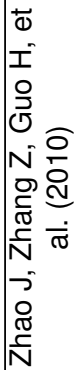 & 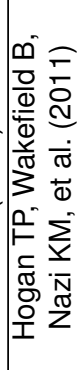 & 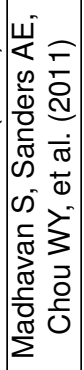 & 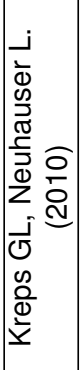 & 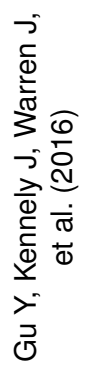 & 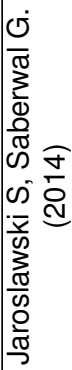 & 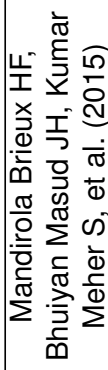 & 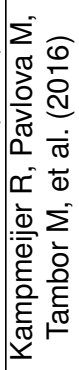 & 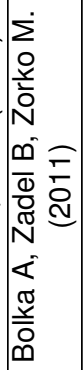 & 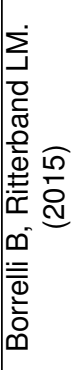 & 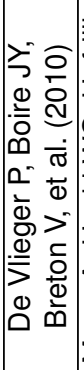 & 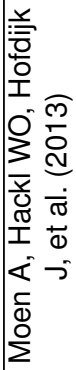 \\
\hline 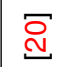 & $\overline{\bar{\Sigma}}$ & $\stackrel{\mathbb{N}}{\mathbb{N}}$ & న్ల & $\underset{\stackrel{\mathbb{U}}{\mathrm{I}}}{ }$ & $\stackrel{\mathbb{N}}{\mathbb{N}}$ & $\underset{\mathbb{N}}{\mathbb{N}}$ & $\stackrel{\widehat{N}}{\underline{N}}$ & 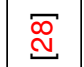 & న్న & 몰 & $\overline{\bar{m}}$ & $\underset{\widetilde{d}}{\widetilde{N}}$ & ल्ల్ల & 窇 & 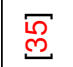 & $\begin{array}{l}\bar{\Phi} \\
\stackrel{\infty}{ }\end{array}$ & 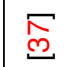 \\
\hline
\end{tabular}




\begin{tabular}{|c|c|c|c|c|c|c|c|c|c|c|c|c|c|}
\hline$\times$ & $\times$ & & & & $\times$ & & $\times$ & & & $\times$ & $\times$ & $\times$ & $\times$ \\
\hline \multirow[t]{7}{*}{$\times$} & & & & & & $\times$ & $\times$ & $\times$ & $\times$ & $\times$ & $\times$ & $\times$ & $\times$ \\
\hline & $\times$ & $\times$ & & $\times$ & $\times$ & & & & $\times$ & $\times$ & & & \\
\hline & $\times$ & $\times$ & $\times$ & & & & & $\times$ & $\times$ & $\times$ & & $\times$ & $\times$ \\
\hline & & $\times$ & & $x$ & & & & & & & $\times$ & $\times$ & $\times$ \\
\hline & & & & & & & & & & $\times$ & & & \\
\hline & & & & & & & & & & $\times$ & & & \\
\hline & $\times$ & $\times$ & & $\times$ & $\times$ & & $\times$ & & $\times$ & $\times$ & $\times$ & $\times$ & $\times$ \\
\hline$\times$ & $\times$ & & $\times$ & $\times$ & $\times$ & $\times$ & $\times$ & $\times$ & $\times$ & $\times$ & $\times$ & & \\
\hline 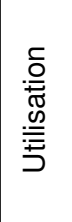 & 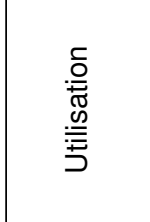 & 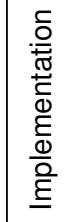 & 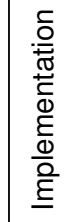 & 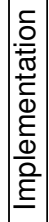 & 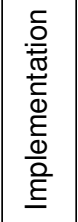 & 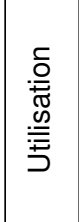 & 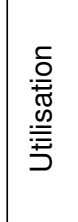 & 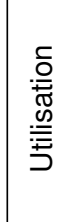 & 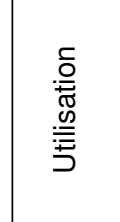 & 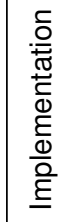 & $\stackrel{\check{E}}{\overline{0}}$ & 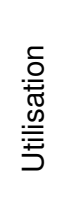 & 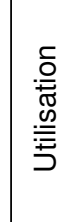 \\
\hline 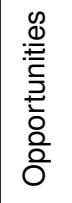 & 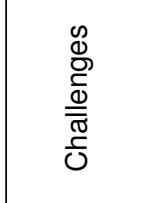 & 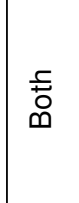 & 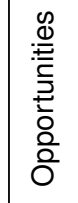 & 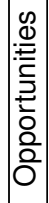 & 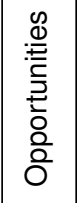 & 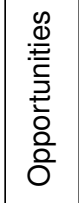 & 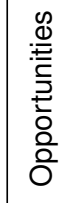 & $\begin{array}{l}\text { 동 } \\
\text { D }\end{array}$ & 돔 & 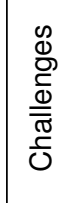 & 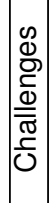 & 劳 & $\begin{array}{l}\text { Fे } \\
\text { D }\end{array}$ \\
\hline$\frac{\xi}{\bar{D}}$ & $\frac{\overline{\bar{N}}}{\frac{\mathbb{N}}{\bar{\infty}}}$ & 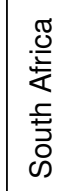 & 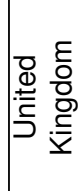 & 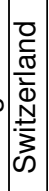 & 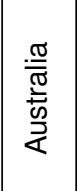 & 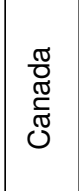 & 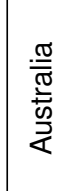 & $\begin{array}{l}\frac{1}{0} \\
\frac{0}{0} \\
3 \\
\text { क }\end{array}$ & 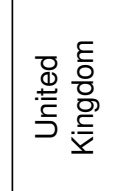 & 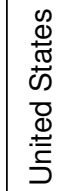 & 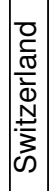 & $\begin{array}{l}\frac{1}{0} \\
\frac{0}{0} \\
\vdots \\
0\end{array}$ & $\frac{\frac{c}{0}}{\frac{D}{\sigma}}$ \\
\hline 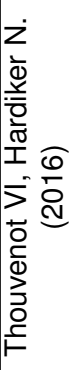 & 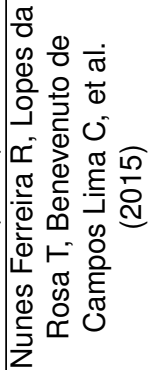 & 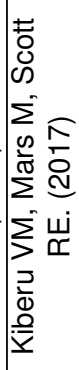 & 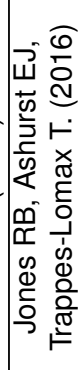 & 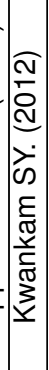 & 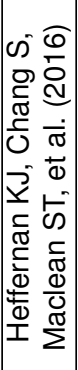 & 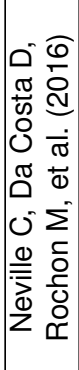 & 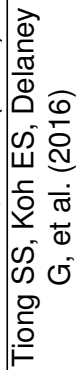 & 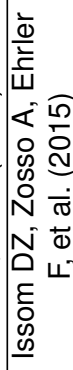 & 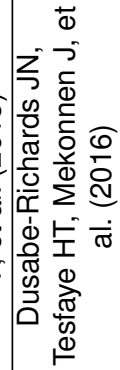 & 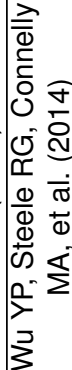 & 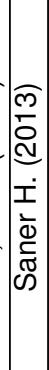 & 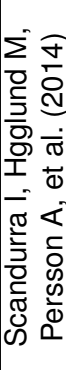 & 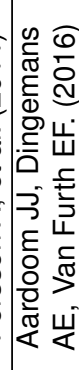 \\
\hline 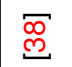 & क্ল & 定 & 焉 & 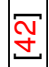 & $\stackrel{\text { g }}{\dot{g}}$ & 步 & 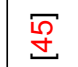 & $\underset{\square}{\mathscr{Q}}$ & 守 & 孪 & 宁 & 믐 & $\overline{\underline{n}}$ \\
\hline
\end{tabular}




\section{Challenges}

The identified challenges to the implementation and utilisation of eHealth are as follows:

C1: Stakeholders and System Users - The functional and non-functional requirements along with the best interests of individual stakeholders should be better integrated into the design and implementation of eHealth applications. There is a need for active involvement and collaboration from all those involved; therefore, requiring the need for common understanding between all those concerned as well as combined participation and familiarity with associated processes [5]. For eHealth to progress, key factors relating to the end-user such as their attitudes [6], [7], [8] and their education [9], [10], [11], [12] prior to implementing an eHealth service should be considered. However, additional factors such as the level of training provided to an end-user [7], [9], [13] after an eHealth service has been implemented should also be considered. Further barriers such as negative user engagement and technological disconnect [14], [15], [16], [17] need to be measured and can be overcome by putting greater importance on the development of new user skills as well as uniting such ideas with organisational changes e.g. providing regular on-thejob training (theory and practical). The examined literature consequently emphasises the significance of the role of end-users and therefore highlights the importance of user-centred design, i.e. to develop an improved appreciation of the design and development process from the users point of view and to focus on developing a detailed representation of the users perspective [18].

C2: Technology and Interoperability - Challenges surrounding integration/interoperability [19], [20], [21], [22], [23], [24] and standardisation of technologies/lack of data standards [9], [25], [26] were two barriers that appeared often in the analysed set of literature. Interoperability should allow for the unified integration of varied technologies to enable secure and fast access to health data and to patient information situated in different locations. However, the barriers identified also suggest a need for the standardisation of system components and services so that healthcare providers can avoid issues such as lack of data standards permitting the exchange of clinical data [26]. One such recent technology that can be used to overcome such hurdles is FHIR (Fast Healthcare Interoperability Resources). FHIR is the latest standard developed by HL7, which describes data formats and elements known as resources and provides users an API (Application Programming Interface) for the exchange of electronic health records. The literature also states that despite the availability of eHealth interoperability, data standards adoption has been slow and requires more effort and commitment to progress its growth. Barriers such as user-friendliness, userinterfaces [7], [27] and legacy systems [21], which still play an integral role in everyday healthcare service delivery, contribute to this problem. This highlights the discrepancy and potential of developing interoperable and standardised technologies [28] that facilitate developing user-friendly, engaging and easily accessible eHealth applications [29].

C3: Cost-Effectiveness and Sustainability - The availability of funding [30], [31] and other resources [11], [32] directly contribute to the impact of the other identified categories of challenges as well as on how well implemented a solution is in practice. Much of the costs are associated with the development of eHealth services/technologies throughout the System Development Life Cycle (SDLC) such as the costs associated with deployment and training [9] as well as the costs for ongoing maintenance of the service/technology in question. A lack of sustained and sufficient funding from stakeholders such as governing bodies e.g. the government [33] indirectly affect the quality of service that healthcare providers can provide to their patients. A further problem, agreeing on reimbursement [24], can be a challenge for new technologies especially as they are of unproven value [7] as well as when some technologies are initially viewed as too expensive to integrate into everyday clinical practice [31]. The literature, 
therefore highlights the need to improve cost-effectiveness and avoid initiatives, which are considered as wasteful [32]. The literature also recommends that future eHealth programs should demonstrate the cost-effectiveness of eHealth programs [25]. A further recommendation is for new initiatives to invest in developing areas of eHealth and emerging trends such as in the field of social computing, deep learning and Internet of Things (IoT).

C4: Legal Clarity and Legal Framework Another major area of challenges revolves around the legalities and legislation involved when developing and introducing an eHealth solution into everyday clinical practice. Legal challenges [9], [21], [34] such as the lack of a legal framework can limit end-user confidence and needs to be addressed prior to any implementation. Legal barriers appear to emerge over time and with the growing use of eHealth technologies and services, make it a challenge to predict and prevent unforeseen legal barriers. There is also the responsibility of adapting to changing legal barriers e.g. when a law has been updated/amended. In terms of security, the obligation for confidentiality [7] and clarity around health data ownership [23], [35] indicate these should be considered carefully and specified in the documentation accompanying the design and implementation of an eHealth application. The literature discusses how end-users prefer using tools and services, whilst safe in the knowledge that their privacy is being protected and is not being compromised [9], [23], [36]. However, a grey area that has not been discussed or elaborated upon by the analysed set of literature, which requires further examining, is the concern of liability. An example of liability is in the case of unwanted events such as technical failures or other circumstances that results in severe harm to a patient and/or to their personal information.

C5: Organisational Context and Regional Differences - The final key barrier to note from the literature is that of challenges that arise from within the local environment and challenges that affect different demographics. The analysed literature reveals that first world citizens have comparatively more wealth and resultantly better education [22] whereas the developing world is often faced with hurdles such as having a larger population [37] and larger geographical areas to cover [11], [37] as well as having slower internet adoption rates. In terms of population health, health inequalities also exist between urban and rural areas in less developed countries [11], [37], [38]. However, a problem that exists globally is that even within regions, there can be challenges due to local organisational context [6], [15], [37], [39]. Different hospitals and healthcare centres, for example, adopt and practice their own rules and regulations [11]. These establishments are also tied to organisational bureaucracy, human resources [6], various unconnected ICT systems/services [11] and organisational workflow support [20]. One key message from the literature suggests that many organisations fail to acknowledge that many barriers to the implementation and utilisation of eHealth lie and emerge from within the organisation itself [40]. For eHealth to advance healthcare services in this area, the literature recommends that eHealth applications could potentially be implemented at a level above existing organisation-centred and process-controlled systems.

\section{Opportunities}

The identified opportunities for the implementation and utilisation of eHealth are as follows:

O1: Participation and Contribution - Participation and Contribution signify the need for communities and backing stakeholders (both internal and external) to participate in active policy forums and knowledge generation [15]. Not only will this help stakeholders to understand each others viewpoints but also establish a shared platform between one another. Within this category key areas of discussion and interest 
as stated in the literature include identifying funding/resourcing, [32], agreeing upon policy/practice [7], [32], [37], establishing standardisation [7], [19], [32] and data sharing. Another area of interest is the research into improving and maximising access to healthcare data and relevant health information through online and [41] IT services [14]. As mentioned previously, one way this could be tackled is by using standards such as HL7's FHIR. The literature recommends that discussion and development efforts will need to take place throughout several scheduled and recorded meetings, therefore allowing all stakeholders sufficient time to come to mutual understandings and common agreements. Further discussion areas include recognising and addressing the factors that affect the healthcare system from a governing perspective and clarifying where eHealth fits into the continuum of care [7], [15]. There also seems to be a great need to develop eHealth policies and strategies on a national level, which can be addressed by forming special bodies responsible for developing an integrated plan for the implementation of eHealth initiatives [6].

O2: Foundations and Sustainability - Foundations and Sustainability relate to all the work surrounding building the initial infrastructure of any eHealth project. It also relates to the establishing of IT architectures/services as well as targeting appropriate design and delivery of eHealth projects [14]. Within this category, key areas surrounding foundation include connectivity, [14], [19], [32], health centre infrastructure [22], [42] infrastructure management [7], eHealth network [20], [22] and shared services and integration [20], [22]. There is also a need to improve and enhance information security and privacy, along with the development of relevant laws and regulatory frameworks. Stakeholders of eHealth projects should also consider how they would overcome challenges that arise due to barriers associated with liability. A gap identified from the literature was the need to make more data available for secondary use such as research and the development of more uniform guidelines [43]. Further gaps also highlighted the need to improve existing IT architecture and the usability of IT systems themselves. In terms of sustainability, an imperative need here was to create and achieve economies of scale, to ensure both combined and local efforts are long-lived [7]. By doing so it is intended that this will help stakeholders to see the implications of any key decisions they make, especially decisions that would influence the direction of growth of an eHealth project.

O3: Improvement and Productivity - Improvement and Productivity relate to the service delivery provided to the public, more specifically, to opportunities where eHealth offers a supplemental or improvement to healthcare services. Thus, maximising the benefits of these existing services. The emphasis is on improving the efficiency and effectiveness of eHealth solutions and healthcare services [44], [45], [46] along with the responsibility to develop process support and change management. The argument here is that there are already enough eHealth solutions in use in everyday practice, but how can we improve upon these solutions so that they are being utilised well into the long term [47]. Within this category, the areas of interest here include alternate service delivery models [35], decision support [29], telehealth/telemedicine [29] and supported peer collaboration. The literature also indicates that many systems fail to fulfil security, patient privacy, data ownership [38] and data quality requirements [48], highlighting these and other similar areas that need to be considered and addressed before the implementation of any eHealth project. Researchers and developers also need to learn how to apply these eHealth tools and services fully in order to extend their ability to study and influence health behaviour as well as engage patients [44] [49]. They also need to ensure that these solutions achieve the intended effects that they were set out to achieve during the inception of the project [50]. 
O4: Identification and Implementation - Identification and Implementation relates to the recognition of eHealth applications and subsequent implementation into routine practice. Clinical Decision Support Systems (CDSSs) and community appropriate information management solutions for patients and the public are two examples of where information processing can contribute to better models of care. In this category, key areas of interest include exploiting the use of Electronic Medical/Health Records (EMR/HER) [36], public health information [12], [16], administrative and information systems [20], [25] and performance monitoring and benefits evaluation, [17], [22]. According to the literature, the introduction of new solutions into daily healthcare practice is still lacking or missing [20], [22]. Other literature, instead argues that there are too many solutions in practice, regardless of whether these solutions deliver the intended results or not. Implementing solutions listed in the above key areas of interest could narrow this gap as well as open further opportunities for novel and innovative solutions. The literature also highlights the fact that many solutions either fail to offer ease of use or are not considered affordable everyday solutions. Therefore, supporting a growing demand for original eHealth solutions, that are easy-to-use and good value but also provide users with a range of societal benefits [29] as well as calls for solutions that lie at the heart of exploiting the potential of ICT in order to improve public health.

\section{Conclusion}

To appreciate the benefits of eHealth, the challenges mentioned in this paper must be overcome or at least reduced to a more manageable outcome. By confronting these challenges, this increases eHealth's potential for rapid growth whether it is via the introduction of new solutions or through the improvement of existing ones. As established, many of the papers identified management of human resources along with skills in relation to information systems design and implementation as huge areas that are crucial to the successful application of eHealth within the health sector. Before eHealth can fully transform health, there must be improved reliability and robustness with the aim of realising more long-term benefits going forward. Governments and national bodies should ensure that eHealth becomes an integral part of all health organisations. They should also take the initiative to build competencies for eHealth as well as find and retain skilled/knowledgeable individuals. These individuals should continually seek to discover new and overcome the existing challenges as well as seize the opportunities mentioned in this paper. Upon deployment of eHealth solutions, stakeholders need to ask themselves have the eHealth initiatives they have implemented made enough efforts in uniting the health community by examining means of providing the correct foundations as well as ask have these initiatives provided suitable access to knowledge suited to the health requirements of all those concerned.

A major limitation of this study is due to the rapid advancement of medical research and technology, which forces researchers to update their findings constantly to avoid publishing outdated information. Conversely, this limitation was also one of the motivations behind the justification of this review. However, it is acknowledged that this review in time would also need to be updated to accommodate future changes in the field. A further limitation of this study was that it did not account for the biases of authors, therefore the identification of challenges and opportunities by these authors could have been influenced by unknown reasons. It is recommended that future papers in the field take into consideration the bias of authors in their works. Nonetheless, this paper identified challenges to implementing eHealth solutions that cut across several categories. Unsurprisingly, the most prominent challenges to eHealth implementation appear to be technology and human-related and it is largely because the core of eHealth 
lies in the interaction between a person and a technology. Within these two categories, the two biggest opportunity areas identified were:

(i) The delivery and participation of stakeholders in active policy forums and knowledge generation, especially to analyse and design how an eHealth solution influences routine clinical practice and healthcare service delivery.

(ii) The introduction of new and novel eHealth solutions that make use of existing and proven technologies such as clinical decision support systems and community appropriate information management solutions.

It follows that the development of future eHealth based projects would benefit most from close collaboration of healthcare professionals with IT specialists throughout, as these projects demand equal awareness and engagement from experts in both fields. However, many of the literature analysed, highlight the requests of these professionals for further research to bring the field of eHealth forward and to determine its place and identity within the healthcare service delivery environment. The literature analysed also address the fact that the potential for improving health outcomes through innovative methods of ICT and eHealth utilisation is vast. Solutions must not only aim to solve current problems but also anticipate future needs, therefore, stakeholders need to address where the solution will be and the impact it will have 5 to 10 years down the line in order to facilitate the best long-term outcomes. One potential way future solutions can benefit substantially is if they can build on prior studies and technologies to measure the current effects that they are having in practice today. Developing tools and systems that release the maximum potential of combining the unique skills of humans and ICT systems will surely revolutionise public health. This review provides a framework to aid in the identification of opportunities to take eHealth implementation to a new level by overcoming recognised challenges. Future work will, therefore, focus on the realisation of the above opportunities.

\section{Declaration of conflicting interests}

The author(s) declared no potential conflicts of interest with respect to the research, authorship, and/or publication of this article.

\section{Funding}

The author(s) declared no financial support with respect to the research, authorship, and/or publication of this article.

\section{References}

[1] WHO. Global diffusion of eHealth: making universal health coverage achievable. Report of the third global survey on eHealth. Global Observatory for eHealth, Geneva: World Health Organization, 2016.

[2] NIB. Using Data and Technology to Transform Outcomes for Patients and Citizens: a framework for action. Personalised Health and Care 2020, London: National Information Board, 2014.

[3] WHO. Health 2020. A European policy framework and strategy for the 21st century (2013). Health 2020: the European policy for health and well-being, Copenhagen: WHO Regional Office for Europe, 2013. 
[4] NHS Digital. Fit for 2020 - Report from the NHS Digital Capability Review. Modernising our delivery model, Leeds: NHS Digital, 2017.

[5] Molleda L, Bahamon M, St George SM et al. Clinic Personnel, Facilitator, and Parent Perspectives of eHealth Familias Unidas in Primary Care. J Pediatr Health Care 2017; 31(3): 350-361.

[6] Alsulame K, Khalifa M and Househ M. eHealth in Saudi Arabia: Current Trends, Challenges and Recommendations. Stud Health Technol Inform 2015; 213: 233-236.

[7] Moore Z, Angel D, Bjerregaard J et al. Chapter 5: Barriers and facilitators for eHealth. J Wound Care 2015; 24(Suppl 5): s25-s31.

[8] Landis-Lewis Z, Manjomo R, Gadabu OJ et al. Barriers to using eHealth data for clinical performance feedback in Malawi: A case study. Int J Med Inform 2015; 84(10): 868-875.

[9] Sharifi M, Ayat M, Jahanbakhsh M et al. E-health implementation challenges in Iranian medical centers: a qualitative study in Iran. Telemed J E Health 2013; 19(2): 122-128.

[10] Devriendt E, Wellens NI, Flamaing J et al. The interRAI Acute Care instrument incorporated in an eHealth system for standardized and web-based geriatric assessment: strengths, weaknesses, opportunities and threats in the acute hospital setting. BMC Geriatr 2013; 13(1): 90.

[11] Henriquez-Camacho C, Losa J, Miranda JJ et al. Addressing healthy aging populations in developing countries: unlocking the opportunity of eHealth and mHealth. Emerg Themes Epidemiol 2014; 11(1): 136 .

[12] Zulman DM, Jenchura EC, Cohen DM et al. How Can eHealth Technology Address Challenges Related to Multimorbidity? Perspectives from Patients with Multiple Chronic Conditions. J Gen Intern Med 2015; 30(8): 1063-1070.

[13] Chan AH, Reddel HK, Apter A et al. Adherence Monitoring and E-Health: How Clinicians and Researchers Can Use Technology to Promote Inhaler Adherence for Asthma. J Allergy Clin Immunol Pract 2013; 1(5): 446-454.

[14] Hardiker NR and Grant MJ. Barriers and facilitators that affect public engagement with eHealth services. Stud Health Technol Inform 2010; 160(Pt 1): 13-17.

[15] Mair FS, May C, O'Donnell C et al. Factors that promote or inhibit the implementation of e-health systems: an explanatory systematic review. Bull World Health Organ 2012; 90(5): 357-364.

[16] Christensen H, Reynolds J and Griffiths KM. The use of e-health applications for anxiety and depression in young people: challenges and solutions. Early Interv Psychiatry 2011; 5(Suppl 1): $58-62$.

[17] Griebel L, Sedlmayr B, Prokosch HU et al. Key factors for a successful implementation of personalized e-health services. Stud Health Technol Inform 2013; 192: 965.

[18] Petersen LS, Bertelsen P and Bjørnes C. Cooperation and communication challenges in small-scale eHealth development projects. Int J Med Inform 2013; 82(12): e375-e385. 
[19] Alkhaldi B, Sahama T, Huxley C et al. Barriers to implementing eHealth: a multi-dimensional perspective. Stud Health Technol Inform 2014; 205: 875-879.

[20] Gusew N, Gerlach A, Bartkiewicz T et al. eHealth vision towards cooperative patient care-domain fields and architectural challenges of regional health care networks. Stud Health Technol Inform 2010; 160(Pt 1): 386-390.

[21] Kluge EH. e-Health promises and challenges: some ethical considerations. Stud Health Technol Inform 2011; 164: 148-153.

[22] Gusew N, Bartkiewicz T, Bautsch W et al. A Regional Health Care Network: eHealth.Braunschweig Domain Fields and Architectural Challenges. Methods Inf Med 2012; 51(3): 199-209.

[23] Kouri P. No turning back - prospects and challenges of eHealth. World Hosp Health Serv 2015; 51(3): 20-24.

[24] Hollmark M, Lefevre Skjöldebrand A, Andersson C et al. Technology Ready to be Launched, but is there a Payer? Challenges for Implementing eHealth in Sweden. Stud Health Technol Inform 2015; 211: 57-68.

[25] Zhao J, Zhang Z, Guo H et al. E-health-Oriented Community Health Information System in China: Our Challenges, Solution, and Experience. Telemed J E Health 2011; 17(7): 584-588.

[26] Zhao J, Zhang Z, Guo H et al. E-health in China: challenges, initial directions, and experience. Telemed J E Health 2010; 16(3): 344-349.

[27] Hogan TP, Wakefield B, Nazi KM et al. Promoting access through complementary eHealth technologies: recommendations for VA's Home Telehealth and personal health record programs. J Gen Intern Med 2011; 26(Suppl 2): 628-635.

[28] Madhavan S, Sanders AE, Chou WY et al. Pediatric palliative care and eHealth opportunities for patient-centered care. Am J Prev Med 2011; 40(5 Suppl 2): s208-s216.

[29] Kreps GL and Neuhauser L. New directions in eHealth communication: opportunities and challenges. Patient Educ Couns 2010; 78(3): 329-336.

[30] Gu Y, Kennely J, Warren J et al. Identifying eHealth Opportunities to Support Medication Adherence - Findings of a Focus Group Study. Stud Health Technol Inform 2016; 223: 150-157.

[31] Jarosławski S and Saberwal G. In eHealth in India today, the nature of work, the challenges and the finances: an interview-based study. BMC Med Inform Decis Mak 2014; 14(1): 1-12.

[32] Mandirola Brieux HF, Bhuiyan Masud JH, Kumar Meher S et al. Challenges and Hurdles of eHealth Implementation in Developing Countries. Stud Health Technol Inform 2015; 216: 434-437.

[33] Kampmeijer R, Pavlova M, Tambor M et al. The use of e-health and m-health tools in health promotion and primary prevention among older adults: a systematic literature review. BMC Health Serv Res 2016; 16(Suppl 5): 290. 
[34] Bolka A, Zadel B and Zorko M. Personal information protection - exceptional challenges of integrated systems of eHealth. Stud Health Technol Inform 2011; 165: 74-79.

[35] Borrelli B and Ritterband LM. Special issue on eHealth and mHealth: Challenges and future directions for assessment, treatment, and dissemination. Health Psychol 2015; 34(Suppl): 12051208 .

[36] De Vlieger P, Boire JY, Breton V et al. Sentinel e-health network on grid: developments and challenges. Stud Health Technol Inform 2010; 159: 134-145.

[37] Moen A, Hackl WO, Hofdijk J et al. eHealth in Europe - Status and Challenges. Yearb Med Inform 2013; 8: 59-63.

[38] Thouvenot VI and Hardiker N. Facilitating eHealth for All Through Connecting Nurses and the Women Observatory for eHealth. Stud Health Technol Inform 2016; 225: 777-779.

[39] Nunes Ferreira R, Lopes da Rosa T, Benevenuto de Campos Lima C et al. The Challenge of e-Health Presence on a Petroleum Platform: Using Telemedicine to Make Operation of Pre-Salt Wells a Reality. Stud Health Technol Inform 2015; 216: 937.

[40] Kiberu VM, Mars M and Scott RE. Barriers and opportunities to implementation of sustainable e-Health programmes in Uganda: A literature review. Afr J Prim Health Care Fam Med 2017; 9(1): e1-e10.

[41] Jones RB, Ashurst EJ and Trappes-Lomax T. Searching for a sustainable process of service user and health professional online discussions to facilitate the implementation of e-health. Health Informatics $J$ 2016; 22(4): 948-961.

[42] Kwankam SY. Successful partnerships for international collaboration in e-health: the need for organized national infrastructures. Bull World Health Organ 2012; 90(5): 395-397.

[43] Heffernan KJ, Chang S, Maclean ST et al. Guidelines and Recommendations for Developing Interactive eHealth Apps for Complex Messaging in Health Promotion. JMIR mHealth and uHealth 2016; 4(1): e14.

[44] Neville C, Da Costa D, Rochon M et al. Development of the Lupus Interactive Navigator as an Empowering Web-Based eHealth Tool to Facilitate Lupus Management: Users Perspectives on Usability and Acceptability. JMIR Res Protoc 2016; 5(2): e44.

[45] Tiong SS, Koh ES, Delaney G et al. An e-health strategy to facilitate care of breast cancer survivors: A pilot study. Asia Pac J Clin Oncol 2016; 12(2): 181-187.

[46] Issom DZ, Zosso A, Ehrler F et al. Exploring the Challenges and Opportunities of eHealth Tools for Patients with Sickle Cell Disease. Stud Health Technol Inform 2015; 216: 898.

[47] Dusabe-Richards JN, Tesfaye HT, Mekonnen J et al. Women health extension workers: Capacities, opportunities and challenges to use eHealth to strengthen equitable health systems in Southern Ethiopia. Can J Public Health 2016; 107(4/5): e355-e361. 
[48] Wu YP, Steele RG, Connelly MA et al. Commentary: Pediatric eHealth Interventions: Common Challenges During Development, Implementation, and Dissemination. J Pediatr Psychol 2014; 39(6): 612-623.

[49] Saner H. eHealth and telemedicine: current situation and future challenges. Eur J Prev Cardiol 2013; 20(2 Suppl): $1-2$.

[50] Scandurra I, Hägglund M, Persson A et al. Disturbing or Facilitating? - On the Usability of Swedish eHealth Systems 2013. Stud Health Technol Inform 2014; 205: 221-225.

[51] Aardoom JJ, Dingemans AE and Van Furth EF. E-Health Interventions for Eating Disorders: Emerging Findings, Issues, and Opportunities. Curr Psychiatry Rep 2016; 18(4): 42. 